\begin{tabular}{ll}
\hline Endless flexibility & 830 \\
\hline Driving fast & 830 \\
\hline Mix and match & 832 \\
\hline Habits die hard & 832 \\
\hline Box 1: Business models for new reagents & 831
\end{tabular}

\title{
Calling the next generation of affinity reagents
}

\section{As complements to antibodies, new reagents to target proteins invite broad types of experiments.}

If only reagents such as antibodies were straightforward to make, stable for many years, versatile, renewable and cheap and could let scientists query scores of proteins at once. These may sound like biochemistry daydreams, but they are promises from researchers working on new types of affinity reagents, including recombinant and engineered antibodies and nucleic acid aptamers.

These tools to lasso proteins add to an expansive menu of polyclonal antibodies, which are generated in animals, and monoclonal antibodies, which are generated in cell lines. Both were the subject of last month's technology feature ${ }^{1}$. The new reagents do not magically dispel the need for validation. Time spent characterizing them will pay off, says Aled Edwards, proteomics researcher at the University of Toronto. From year to year the reagents are "the exact same thing," he says. Researchers can accrue and compare data over time.

Design flexibility could give these reagents superiority over a polyclonal or a monoclonal, says Edwards. "For the recombinant ones you can do all sorts of things, put little tags on them, and little hooks and bells and whistles," he says. "They're more versatile, for sure."

He says that he hopes the availability of these reagents will entice scientists to cast a wider net-beyond one protein to a family of proteins, for example. "It's a little bit risky because it takes longer to come to the answers." But scientists now take risks on antibodies that might not be fully characterized. "Renewables will be a better reagent in the long run, without question," he says.

\section{Creating choices}

More choice also means more time spent choosing: selecting one reagent over another or even modifying protocols.
Scientists in two

large research projects and some companies, too, bring forward the reasons these options are a good idea. They are developing new reagents and highthroughput ways to generate them.

Money is one reason. A lab can obtain a set of monoclonal anti-

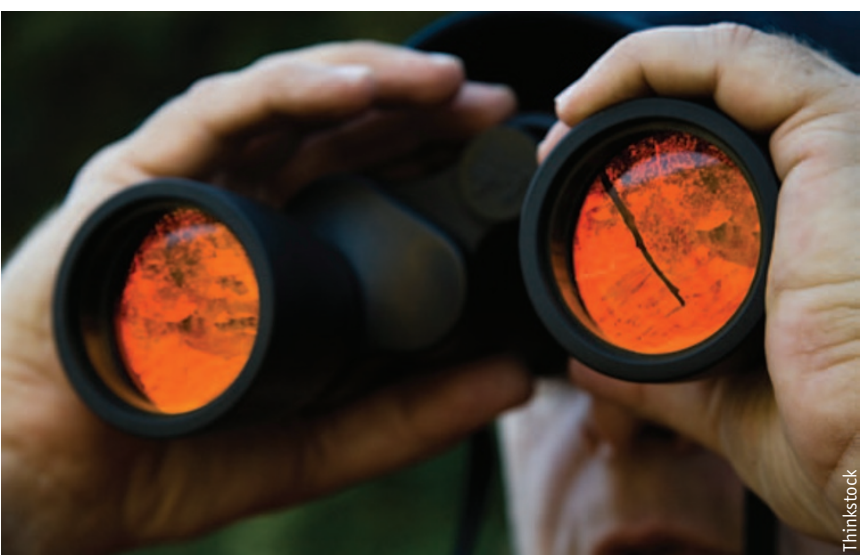

New reagent types are on the horizon.

bodies from a service provider by supplying antigens for the proteins of interest. But if scientists want to investigate 500 or even 1,000 proteins or look at a pathway involving 40 proteins, that approach will break the bank in most labs, says Salvatore Sechi, program manager of the US National Institutes of Health (NIH) Common Fund's \$54-million Protein Capture Reagents program, now in its second year.

Two NIH reagent production centers are targeting $\sim 1,500$ human transcription factors, many of which have a role in disease. As Sechi explains, no renewable affinity reagents exist for most of these proteins. One project, at Johns Hopkins University, is building a pipeline for monoclonal antibodies, and the other, at the University of Chicago, is focused on generating recombinant antibodies. Both groups are addressing some underestimated production bottlenecks, Sechi says. "The production of the antigen is definitely one of the major challenges in this field." For many applications antigens need to be properly folded, a requirement that is both technically and financially challenging to meet.

The two centers will generate validated reagents for human transcription factors to be made available through community resources such as the University of Iowa's Developmental Studies Hybridoma Bank and DNASU at Arizona State University. Separate projects are geared toward improving present technology.

The four US technology hubs developing high-throughput approaches to generating new reagents are at Los Alamos National Laboratory, Arizona State University, the University of Illinois at Chicago and the University of California, Santa Barbara. These centers have cooperative arrangements with the NIH that involve closer interaction than the traditional funding mechanisms and include ongoing evaluation by external panels, Sechi says.

In the EU affinity-reagents project, technology development and reagent generation are combined. "We've concentrated on establishing the pipeline, whereas NIH also has these technology centers which are looking very much to the future," says Michael Taussig of the UK Babraham Institute, who coordinates the \$20-million EU program with 20 teams across Europe. Affinomics is the third phase of the multi-year, EU-financed program focused on new reagents for signaling 


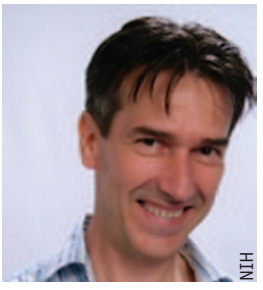

Antigen production is one of the field's major challenges, says Salvatore Sechi. molecules, another class of targets not comprehensively served by commercial offerings. "We purposefully avoided competing in terms of the targets we choose," Taussig says.

Some projects, such as the Human

Protein Atlas, look broadly at developing affinity reagents for all human proteins, says Taussig. Other projects look deeply. A binder can be right for a particular receptor, but some scientists might want reagents to bind only when the receptor is activated and has a different conformation. "You can go to quite a lot of depth on any one single protein, which may require lots of different types of reagents," he says.

At present, none of the new affinityreagent types stand head and shoulders above the others. "It might turn out, but we don't know for certain, that there are going to be many winners," Sechi says. One type of reagent might work better for certain applications, such as western blotting or immunoprecipitation. As the teams swap ideas across the Atlantic and encounter similar roadblocks, communication may pave the way for a joint effort. "We would like it to become an international project," Taussig says.

\section{In the trenches}

Both the EU and NIH projects carry a tone of urgency in addressing the needs of researchers such as Karen Colwill, a molecular biologist in Tony Pawson's lab at the Lunenfeld-Tanenbaum Research Institute at Mount Sinai Hospital in Toronto. A few weeks ago, Colwill had a 'moment', and not just because of the rainstorm that was crashing over the city. Flooding her thoughts was an e-mail she had received about an antibody she and her lab colleagues use. The e-mail's subject read: "Antibody discontinued."

The day had started well. Experiments with a polyclonal antibody from a commercial vendor were moving along, helping Colwill to study protein-protein interactions using mass spectrometry. Without any warning, the vendor was now removing the antibody from its catalog.

The good news was that the company had a monoclonal alternative. But after several weeks of testing this monoclonal antibody and eight others, Yong Zheng, a scientist in the lab, came to Colwill with grim news. No other antibody was comparable to the discontinued polyclonal.

The company's monoclonal was much less efficient in protein 'pulldown', in which an antibody is used to latch onto and weigh down a protein of interest, allowing it to be isolated for further study. Compared to the polyclonal, the monoclonal anchored only $10 \%$ as much protein, not enough to probe the particular interaction the lab is studying. The lab members are extremely frustrated. Work has been on hold for the past 2-3 months, she says, as the group hunts for the right affinity reagent. She hopes that recombinant antibodies will soon fill the gap in her situation and others like it. "I think we're at that stage where they're about to," says Colwill.

\section{Endless flexibility}

Recombinant technologies help generate molecular libraries from which protein binders can be selected, using, for example, phage display. Bacteriophages, viruses that attack bacteria, are engineered to express peptides or proteins. "You expose the library to your target and try and select out the ones which you need," Taussig says. The approach offers the tempting prospect of an endless supply of affinity reagents. Monoclonals are, in theory, endless in supply, but they are not always the answer, as Colwill's dilemma shows.

Recombinant antibodies appeal to Colwill also because they are quick to screen, they are generated in vitro and the DNA sequence is available to researchers. "I really hope that this does mature and starts becoming the standard," she says. She collaborates with Sachdev Sidhu of the University of Toronto, who ramps up affinity-reagent production by combining phage display, high-throughput screening and sequencing. With such technology, 100 different recombinant antibodies can be generated to bind an antigen. Presented with such a range of choices, scientists could mix and match reagents for their protocols. "You could say 'my best results are if I use a mixture of antibody 1, 5 and 7', Colwill says.

This type of combination could recapitulate the strength of polyclonals that can bind to different spots on a protein, finding one epitope when another is blocked in some way. This 'polyclonal effect' might

be what is missing from her current monoclonal assay, she says.

Recombinant technology offers the flexibility to engineer the fragment antigenbinding site, which is the variable region of the antibody, she says. Research groups can screen, validate and alter residues to obtain reagents with the affinity traits they seek.

The new reagents might end up with a new look; for example, they could be antibodies with more than two arms. But scientists will be most comfortable using traditional antibodies that come in the Y-shaped format of immunoglobulin $\mathrm{G}(\mathrm{IgG})$, says Colwill. The protocol then would be "business as usual." If a recombinant antibody is modified with a His tag or a biotin tag, it has to be purified slightly differently, she says.

\section{Driving fast}

Generating affinity reagents quickly is an important goal in this field. One approach to cut screening time for recombinant antibodies is to add high-throughput sequencing to techniques such as phage or ribosome display, says Taussig.

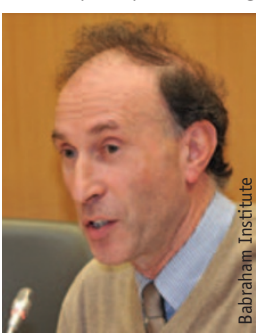
on one protein can require many types of reagents, says Mike Taussig.
Going to depths

The selection of nucleic acid aptamers, which can be done through a process called systematic evolution of ligands by exponential enrichment (SELEX) and which applies chemical synthesis, is time consuming. Hyongsok Tom Soh and his team at the

University of California, Santa Barbara, are using microfluidics to help select aptamers and adding high-throughput sequencing to the workflow. "You could do a lot of selections in parallel, and you could do nextgeneration sequencing to identify what you have selected," Taussig says of this approach.

The advantage of using aptamers is that the reagent is a sequence that can be downloaded from a website, says NIH's Sechi. Nucleic acid protein avimers, or NuPromers, are another type of e-mailable reagent that Arizona State University biochemist John Chaput and his team are developing. These reagents can recapitulate antibody affinity and specificity, says Chaput. 


\section{BOX 1 BUSINESS MODELS FOR NEW REAGENTS}

A number of companies are eager to help make new affinity reagents commercially successful. Some are or have been involved with the NIH and EU-funded projects and, rather than sell individual reagents, they offer assays.

Ulf Landegren, who is part of the EU-funded Affinomics, co-founded Olink Bioscience, a company that sells assays applying proximity ligation technology. Larry Gold, founder of a company called SomaLogic, calls Landegren's technology an "unbelievably clever" way to measure many proteins at once. The goal of finding informative and specific biomarkers-much like that of detecting the winning lottery tickets hidden in a huge pile of non-winners-is "my life," says Gold.

As an entrepreneur and biology professor at the University of Colorado Boulder, Gold has spent the past two decades developing renewable affinity reagents, and

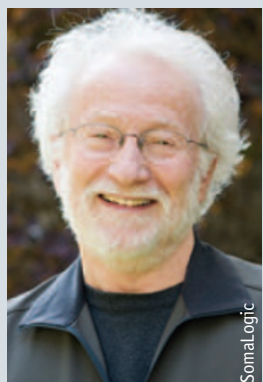

Finding useful biomarkers is "my life," says Larry Gold. they have now matured, he says. Stable, long-term financing from unnamed sources as well as collaborations with pharma companies have helped him to stay the course in developing slow off-rate modified aptamers, or SOMAmers ${ }^{6}$. These DNA oligonucleotides with chemically modified nucleotides are generated by SELEX and engineered to have capabilities such as equilibrium binding and slow off-rates. "Those two things together allow us to have the kind of specificity needed to measure proteins in blood with one reagent instead of two," he says. The approach is a kind of "non-sandwich sandwich', using one reagent instead of the conventional two antibodies.

Standard ELISA assays can detect $\sim 40$ analytes. Gold says his SOMAmer-based approach can now distinguish among and measure $>1,000$ proteins in a drop of blood, and that number climbs in his company's lab. On a fee-for-service basis he offers SOMAscan, a multiplexed assay for proteomic analysis of blood samples, for academics and researchers in drug discovery.

Along the way, Gold has shattered 'Gold's wall', an informal term, named after him, for what happens when scientists try to detect more than 20 proteins in blood at once. Noise drowns out the signal, he says. And blood presents other challenges for researchers seeking to detect a protein in the picomolar range among throngs of proteins such as albumin, which are present in millimolar range.

Gold is betting that academia and drug developers will want to apply his technology in their research. "You win by having the reagents being used in the big labs and some people saying 'Holy moly, this is better data than with whatever we were using yesterday'."

Andreas Plückthun of the University of Zurich has founded a company called Molecular Partners to commercialize his affinity reagents, DARPins. He and his team have found ways to select reagents to 96 proteins in parallel, and faster, cheaper and higher-throughput experiments are coming, he says. He is also a co-founder of MorphoSys, a company devoted to the development of antibodies as reagents and for therapeutics. But it was a challenge to be active in both realms, he says, and in December 2012 MorphoSys sold its reagent-antibody business to Bio-Rad.

More generally, he says that it would be difficult for a reagents company to generate the revenues to hire and keep the staff needed to make these tools. As a result, both of Plückthun's companies are focused on therapeutic applications. As technology development progresses, he thinks that DARPins and other affinity reagents will eventually emerge as cheaper tools that can be made in higher throughput, so that "at the end of the day, more can be done by fewer people."

Government funding is helping him to move his technology beyond the "handcrafted' mode of manufacture. "Otherwise this would have never happened," he says. "It's sort of like you've built one hand-crafted Ferrari, and now you have to convert this to an assembly line, but the Ferrari should still work exactly as perfect as before," he says. "That's the challenge." 
Chemical synthesis is an advantage because it "eliminates a lot of the problems associated with genetically encoded reagents produced in cells," Chaput says. "Eventually we would like NuPromers to be available via DNASU, but we are not quite ready for that now."

Although Colwill has not worked as much with aptamers as she has with recombinant antibodies, she welcomes the diversity of the new reagents. Experiments might show that for certain proteins, aptamers work better than recombinant antibodies. "I don't know if they have to be mutually exclusive," she says. The choice of varying molecular formats does, however, mean that vendors or labs must communicate in reagent protocols whether a reagent has an IgG format, for example, or whether certain types of beads or secondary antibodies are required.

\section{Mix and match}

Ever more architectures for affinity reagents are possible. For example, Ulf Landegren at Uppsala University has developed the proximity ligation assay (PLA), a method for dual recognition of the same protein with two binders ${ }^{2}$. With PLA, antibodies or aptamers are tagged with oligonucleotides. They latch on to different spots on a protein. When the binders are attached to the same target protein and close together, it becomes possible to ligate the oligonucleotides together and amplify the product.

Landegren's group has developed these reagents "to give extreme specific-

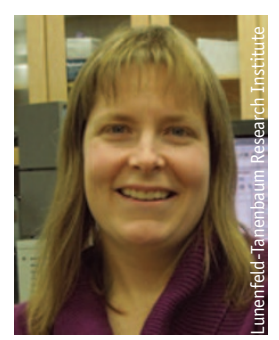

Karen Colwill is interested in recombinant antibodies because they are quick to screen. ity and extreme sensitivity at the same time," says Taussig. Because the approach can work with antibodies or aptamers, one question the team is working on is whether the assays can work with a variety of different binders. "So you could mix and match," he says.

Chaput and others see a day approaching when affinity reagents will be available in large quantities at low cost. On a typically busy day, scientists might appreciate an affinity-reagents portal that offers a wide choice of reagents to mix and match. But they might also struggle with the wealth of choices. "You just want one that works," says Colwill.

Data on affinity and specificity will continue to guide researchers in choosing affinity reagents, Sechi says. Users will still need to do their own validation experiments to see whether reagents will work "in their hands." Specificity continues to be an issue for scientists, Taussig says. Scientists may continue to use 'sandwich' assays, which can involve more than one reagent. "Which of those reagents will work together has not really been worked out," he says. "That's one of the things we're trying to do."

As choice broadens, scientists will want to compare how the reagents perform in diverse assays such as immunoprecipitation or immunohistochemistry. Binding curves are informative, but are not the last word. "There's no gold standard in any of the assays," says Edwards. Scientists tend to say of an antibody, for example, "It's got good staining," he says. But he wishes there were a quick approach to assess antibody quality for a specific application. “There's no quantitative measure, like 'this is an 83-out-of-100 antibody,' he says. Having new affinity reagents, he says, will not solve this basic issue but could make the need to find a solution more pressing.

\section{Habits die hard}

Together with Colwill, Edwards and 49 other scientists built a production-andcharacterization pipeline, generating more than 1,000 monoclonal and recombinant antibodies to 20 proteins containing the Src homology 2 domain ${ }^{3}$. Edwards chose Life Technologies as a distribution platform for these reagents for epigenetics assays. But uptake of the reagents in the research community has been slow, he says. He and others are puzzled.

It takes a while for technology to spread and for labs to test reagents thoroughly, says Colwill. But researchers who are comfortable with certain antibodies do not readily cast them aside. "It's the devil you know," says Edwards. He also points to proteomic favoritism. Along with colleagues at the University of Toronto, the University of North Carolina, Seoul National University and GlaxoSmithKline, Edwards tabulated 20 million papers published between 1950 and 2009. They found that three-quarters of protein research focuses on the $10 \%$ of proteins that were known before the 


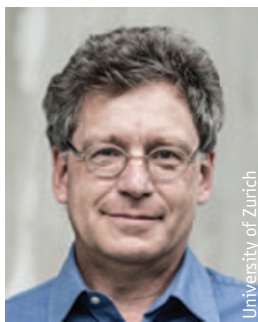

New affinity reagents can be produced on demand and quickly, says Andreas Plückthun.

mapping of the human genome ${ }^{4}$.

Follow-on analyses have not shown much change, Edwards says. The favorite proteins function in pathways of biomedical interest, but much of the proteome still generates few or no papers. "The balance is probably not right," he says. Researchers stick to reagents they know and to target proteins they know.

Edwards hopes a new reagent can be more than a "new shiny antibody for a chestnut," he says, referring to a well-studied protein. New affinity reagents could let scientists ask different, more expansive questions, of the 'chestnuts' as well as the less-studied proteins. New approaches to distribution might also be needed. Research papers describing the advantages of the new reagents will help. But resources that wait for users to come and get new affinity reagents are not working, he says.

"I think the stockpile idea is flawed, because no one will know in advance what you should be stockpiling," says Andreas Plückthun of the University of Zurich, who makes engineered antibody alternatives called designed ankyrin repeat proteins (DARPins) and is involved with the NIH and EU projects. Instead of stockpiling, he prefers an application-oriented approach, because these new affinity reagents can be produced quickly and on demand. His DARPins can be engineered in many formats, with many types of attachments bound either loosely or rigidly ${ }^{5}$. And they can be used within cells, too, which is difficult to do with antibodies, he says.

Plückthun and his team have created DARPins with attached fluorophores, which light up once the affinity reagent latches on to its target to show, for example, activation of extracellular signal-regulated kinase (ERK) in living cells. "You basically have real-time, real-space information about who is activated when and where," he says.

For his DARPins and other affinity reagents, high-throughput methods are in development, and new business models are emerging (Box 1). He acknowledges the nudging from colleagues asking when the new affinity reagents will be ready and cheap. For now, he says, the sophisticated selection methods call for highly qualified researchers, which makes the reagents expensive. "We're still in research mode trying to develop faster and better methods," he says. In his view, which he shares with many others in this emerging field, new affinity reagents will help biology research move far beyond what has been possible with antibodies.

1. Marx, V. Nat. Methods 10, 703-707 (2013).

2. Fredriksson, S. et al. Nat. Biotechnol. 20, 473477 (2002).

3. Colwill, K., Renewable Protein Binder Working Group \& Gräslund, S. Nat. Methods 8, 551-558 (2011).

4. Edwards, A.M. et al. Nature 470, 163-165 (2011).

5. Gu, G.J. et al. Nat. Biotechnol. 30, 144-152 (2013).

6. Mehan, M.R. et al. PLOS ONE 7, e35157 (2012).

Vivien Marx is technology editor for

Nature and Nature Methods

(v.marx@us.nature.com). 